\title{
Gastric Electrical Stimulation Is an Option for Patients with Refractory Cyclic Vomiting Syndrome
}

\author{
Inderpreet Grover, ${ }^{1}$ Richard Kim, ${ }^{2}$ Danielle C Spree, ${ }^{3}$ Christopher J Lahr, ${ }^{4}$ Archana Kedar, ${ }^{2}$ Shivangi Kothari, ${ }^{3}$ David Fleisher, ${ }^{5}$ and \\ Thomas L Abell ${ }^{2 *}$ \\ ${ }^{1}$ Department of Medicine, University of Mississippi Medical Center, Jackson, MS, USA; ${ }^{2}$ Division of Gastroenterology, Hepatology and Nutrition, \\ Department of Medicine, University of Louisville, KY, USA; ${ }^{3}$ Division of Digestive Diseases, ${ }^{4}$ Department of Surgery, University of Mississippi \\ Medical Center, Jackson, MS, USA; and ${ }^{5}$ Department of Pediatric Gastroenterology, University of Missouri Health Care, Columbia, MO, USA
}

\section{Background/Aims}

Cyclic vomiting syndrome (CVS) is a disabling migraine variant manifesting as severe episodes of nausea and vomiting and often refractory to many therapies. Gastric electrical stimulation (GES), which can reduce nausea and vomiting in gastroparesis, may provide symptomatic relief for drug-refractory CVS. This study assessed the utility GES in reducing the symptoms of CVS and improving the quality of life.

\section{Methods}

A one-year, non-randomized, clinical study was conducted. Eleven consecutive patients with drug refractory, cyclic vomiting syndrome based on Rome III criteria and North American Society for Pediatric Gastroenterology, Hepatology and Nutrition (NASPGHAN), underwent treatment with temporary GES (Temp GES) and permanent GES (Perm GES). Post-treatment follow up was done up to one year after permanent gastric electrical stimulation therapy.

\section{Results}

Total symptom score decreased by $68 \%$ and $40 \%$ after temporary and permanent GES therapies, respectively. Hospital admission events significantly decreased to 1.50 ( \pm 1.00) events from 9.14 ( \pm 7.21 ) annual admissions prior to treatment with permanent GES. Vomiting episodes fell by $83 \%$ post Temp GES and 69\% after Perm GES treatments. Mucosal electrogram values also changed after temporary stimulation.

\section{Conclusions}

In a small group of drug-refractory CVS patients, treatments with temporary and permanent GES significantly reduced the severity of gastrointestinal symptoms and frequency of hospital admissions.

(J Neurogastroenterol Motil 2016;22:643-649)

Key Words

Cyclic vomiting syndrome; Gastric electrical stimulation; Nausea; Vomiting

Received: August 20, 2015 Revised: March 9, 2016 Accepted: March 14, 2016

(a) This is an Open Access article distributed under the terms of the Creative Commons Attribution Non-Commercial License (http:// creativecommons. org/licenses/by-nc/4.0) which permits unrestricted non-commercial use, distribution, and reproduction in any medium, provided the original work is properly cited.

*Correspondence: Thomas L Abell, MD

Division of Gastroenterology, Hepatology and Nutrition, Department of Medicine, University of Louisville, 550 S. Jackson Street, ACB3 A3L15, Louisville, KY 40202, USA

Tel: +1-502-852-6991, Fax: +1-502-852-0846, E-mail: thomas.abell@louisville.edu 


\section{Introduction}

Cyclic vomiting syndrome (CVS), initially described in an early 19 th century report, ${ }^{1,2}$ is characterized by random episodes of intense nausea and vomiting that can last from hours to days, interspersed with symptom-free intervals. A recent increase in review articles and CVS case reports has resulted in the 4 phases of this disorder being well described: a symptom-free interval, a prodromal phase, a vomiting phase, and a recovery phase. ${ }^{3,4}$

The actual prevalence of the disease is unknown. All races, ages, and ethnicities can be affected by CVS, although Caucasians and females appear more susceptible than others. ${ }^{4-6} \mathrm{~A}$ recent case report describing the illness among multiple family members suggests that the disorder may be inherited. The precise pathogenesis of CVS is unknown and likely multifactorial, but it may result from dysfunction of neuro-hormonal pathways responsible for the control of nausea and vomiting.

Symptomatic management to abort or terminate an episode is now the mainstay of treatment, with severe bouts of CVS sometimes necessitating hospitalization to address dehydration and restore homeostasis. The potential role of drugs helpful in aborting the attacks has also been well described, with the range of medications used to treat CVS - sumatriptan, erythromycin, carnitine, propranolol, cyproheptadine, coenzyme Q10, nebivolol, tricyclic antidepressants - thoroughly discussed..$^{7-11}$ However, for some drug-refractory patients, the only recourse has been sedation and a quiet environment.

No studies have been reported that examine the potential for gastric electrical stimulation (GES) as treatment for CVS. GES has been shown in clinical trials from as early as 1992 to be a safe and effective treatment option for the nausea and vomiting of drugrefractory gastroparesis (GP), with long-term studies reporting statistically significant improvement for all GP symptoms. ${ }^{12-16}$ One decade-long study showed that many GP patients either remained symptom-free or continued to experience a significant reduction of symptoms with GES. ${ }^{13}$

In this pilot study, we evaluated the effectiveness of GES for treating nausea and vomiting associated with CVS.

\section{Materials and Methods}

A non-randomized, 1 year, clinical study was conducted from August 2009 to August 2010 in the Emergency Department, Clinics, and Outpatient Clinics of the University of Mississippi Medical Center. Patients were again evaluated during their follow- up visits to clinics after permanent GES (Perm GES) and were also followed up by telephone. Records from electronic chart review were obtained to collect data on hospital visits. Patients were part of a larger study of GES with the approval of the University of Mississippi Institutional Review Board.

For this pilot investigation, enrollment steps with inclusion and exclusion criteria were as follows:

Inclusion criteria:

- Gender: male or female

- Age range: 18 years to 70 years in age

- Patients with CVS who met the criteria as defined by Rome III and North American Society for Pediatric Gastroenterology, Hepatology and Nutrition (NASPGHAN)

- Symptoms of CVS for $\geq 1$ year

- Refractory or intolerant to antiemetic drug classes (antihistamines and phenothiazines, serotonin receptor antagonists, dopamine receptor antagonists)

- Chronic vomiting and/or nausea with 7 or more episodes per week for either symptom irrespective of gastric emptying test (GET) values

- The patient is willing and able to provide informed consent.

- The patient is willing and able to return for required followup visits.

Exclusion criteria:

- Patients $<18$ years or $>70$ years in age

- Patients with an active infection of any kind

- Patients with GP

- Patients who the investigator determines are not candidates for endoscopic procedures

- Women who are pregnant

- Inability or unwillingness to provide informed consent

- Unwilling or unable to return for required follow-up visits and examinations

- Patients who are currently enrolled in another investigation of a medical device or drug

A 5-day course of temporary mucosal GES was provided, ${ }^{13,14}$ and for all patients, was followed by permanent stimulator implantation. ${ }^{12-16}$ Symptoms occurring between initial temporary GES (Temp GES) and Perm GES placement were controlled with antiemetics.

For Temp GES, a temporary cardiac pacing lead is endoscopically inserted as close as possible to the junction of the antrum and the body of the stomach. This temporary lead is screwed into the stomach mucosa, and 3 to 5 endoscopic clips are used to hold the lead in place. This lead is then connected to an external GES de- 
Table 1. Comparison of Burden on Health Care with Gastric Electrical Stimulation Therapy in Cyclic Vomiting Syndrome Patients

\begin{tabular}{lccccc}
\hline & Baseline & Temp GES & $P$-value & Perm GES & $P$-value \\
\hline Number of hospital admission days & $9.14 \pm 7.21$ & $5.0 \pm 5.23$ & 0.344 & $1.5 \pm 1.0$ & 0.069 \\
Number of admissions due to vomiting & $6.19 \pm 2.52$ & $3.25 \pm 3.38$ & 0.118 & $2.25 \pm 1.5$ & 0.018 \\
\hline
\end{tabular}

Temp GES, temporary gastric electrical stimulation; Perm GES, permanent GES.

Table 2. Comparison of Electrogastrogram with Gastric Electrical Stimulation Therapy in Cyclic Vomiting Syndrome Patients

\begin{tabular}{llccccc}
\hline & & Baseline & Temp GES & $P$-value & Perm GES & $P$-value \\
\hline Mucosal electrogastrogram & Frequency $(\mathrm{cpm})$ & $5.81 \pm 2.32$ & $4.64 \pm 1.55$ & 0.233 & - & - \\
& Amplitude $(\mu \mathrm{V})$ & $0.53 \pm 1.09$ & $0.32 \pm 0.37$ & 0.602 & - & - \\
Cutaneous electrogastrogram & Frequency $(\mathrm{cpm})$ & $6.69 \pm 4.57$ & $5.72 \pm 1.82$ & 0.584 & $4.98 \pm 2.21$ & 0.498 \\
& Amplitude $(\mu \mathrm{V})$ & $0.12 \pm 0.07$ & $0.12 \pm 0.09$ & 0.904 & $0.12 \pm 0.06$ & 0.979 \\
\hline
\end{tabular}

Gastric mucosal electrogram performed only during temporary gastric electrical stimulation (Temp GES) implantation.

Perm GES, permanent GES.

vice that can be placed in a shirt pocket, telemetry pouch, etc. ${ }^{17}$ For Perm GES, 2 intramuscular electrodes are inserted by laparotomy or laparoscopy into the muscularis propria of the greater curvature of the stomach roughly $10 \mathrm{~cm}$ proximal to the pylorus, and a neurostimulator is positioned subcutaneously in the abdominal wall. The neurostimulator is positioned subcutaneously in the abdominal wall, typically in the right midquadrant. Possible adverse effects to the procedure include infection of the neurostimulator pocket, pain related to lead perforation of the stomach, and discomfort from migration of the pulse. ${ }^{18}$ Pacing of both Temp and Perm GES patients was the same as that used in GP patients (frequency, $14 \mathrm{~Hz}$; intensity, $5 \mathrm{~mA}$; pulse width, $330 \mu \mathrm{sec}$; cycle $\mathrm{ON}, 0.1$ seconds; cycle OFF, 5.0 seconds)..$^{17,18}$

\section{Primary Outcome Measurements}

All patients were evaluated at baseline for the primary outcome parameters associated with gastrointestinal (GI) symptoms, gastric physiology and electrophysiology, and hospital admissions. To measure GI symptoms, a standardized Likert scale PRO derived tool was used to rate nausea, vomiting, bloating, and total symptom score (0-4 each, maximum 20), as well as health-related quality of life. Health related quality of life was evaluated using the investigator-derived independent outcome measure scores (IDIOMS) assessment. Physiological assessments included Body Mass Index and the 4-hour measure of the GET. Gastric emptying was performed with the technetium labeled solid meal measured for 4 hours. ${ }^{19}$ Hospital admissions prior to GES treatment were also determined at the baseline evaluation (Table 1).

At day 1 (GES ON) and day 5 (GES OFF) of Temp GES,

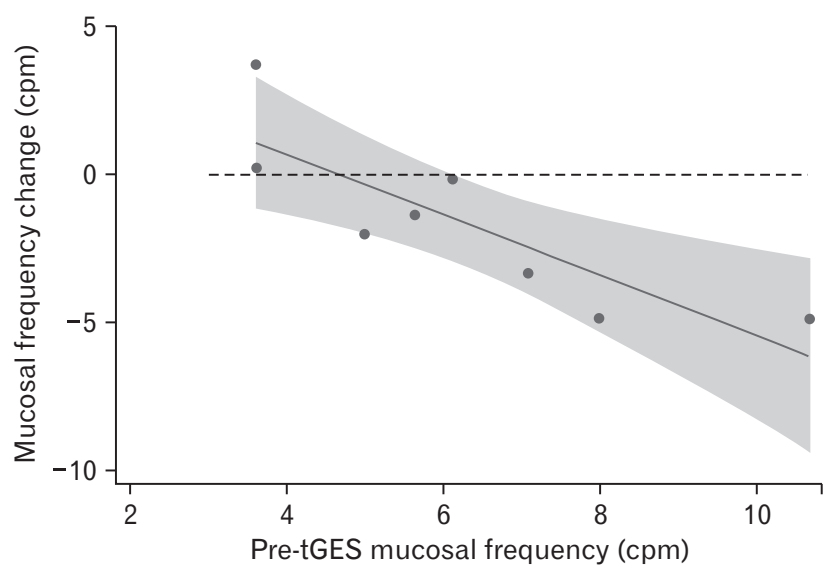

Figure 1. Changes in mucosal frequency vs pre-temporary gastric electrical stimulation (Pre-tGES; baseline) mucosal frequency at temporary GES treatment initiation. Pre stimulation frequency $=4.72$, post stimulation frequency $=4.54$, slope $=-1.02(-1.68,-0.37), P=$ $0.009, \mathrm{R}^{2}=0.71$.

symptom and quality-of-life measures were again obtained, as were the body mass index (BMI) and the 4-hour measure of the GET. Post permanent stimulator placement, symptom and quality of life measures were again collected, along with the BMI and the 4-hour measure of the GET. Cutaneous electrograms as well as mucosal electrograms were obtained at baseline and post-Temp GES. Serosal electrograms were also obtained at the time of Perm GES placement (Table 2 and Fig. 1).

Changes in vomiting scores, total symptom scores, cutaneous electrogram, mucosal electrograms, and 4-hour GET were assessed. Hospital admissions per year were assessed at baseline, at 
Temp GES, and after Perm GES implantation. Follow-up assessments were conducted at 1-year following Perm GES implantation, with patients presenting back to clinic or over the phone. Findings for the primary parameters, as measured at baseline and post temporary and permanent treatment, were compared by paired $t$ tests and reported as mean $\pm \mathrm{SD}$.

\section{Results}

A total of 11 consecutive patients ( 4 white and 3 black males, 4 white females; mean age 38 years; 5 patients with concomitant glu-

Table 3. Demographic Information and Baseline Motility Measures in Patients with Cyclic Vomiting Syndrome ${ }^{a}$

\begin{tabular}{lcr}
\hline \multicolumn{1}{c}{ Variable } & Mean & \pm SD \\
\hline Age & 37.64 & 14.92 \\
Male & $64.00 \%$ & 0.50 \\
Diabetics & $45.00 \%$ & 0.52 \\
White & $73.00 \%$ & 0.47 \\
BMI & 28.96 & 6.99 \\
Nausea & 3.55 & 0.69 \\
Vomiting & 2.68 & 1.27 \\
Bloating & 2.36 & 1.52 \\
Abdominal pain & 2.91 & 1.20 \\
Anorexia/early satiety & 2.55 & 1.44 \\
Total symptom score & 14.05 & 3.23 \\
GET 1 hr & 63.30 & 31.81 \\
GET 2 hr & 41.30 & 28.99 \\
GET 4 hr & 19.90 & 18.08 \\
GET total & 119.44 & 4.34
\end{tabular}

${ }^{a}$ The mean symptom scores for the 5 upper gastrointestinal symptoms associated with cyclic vomiting syndrome (CVS), gastric emptying times, and investigator-derived independent outcome measure scores values at baseline, during temporary mucosal gastric electrical stimulation, and after permanent stimulator implantation for the $11 \mathrm{CVS}$ patients treated with gastric electrical stimulation.

BMI, body mass index; GET, gastric emptying test. cose intolerance), all meeting the Rome III criteria ${ }^{20}$ and NASPGHAN consensus for drug refractory $\mathrm{CVS},{ }^{21}$ were included in our study. Baseline characteristics and motility measures can be seen in Table 3.

Patients were followed after initial Temp GES, which resulted in improvement in nausea and vomiting. These findings were significant and led to Perm GES placement. Patients with CVS treated with GES showed significant improvement in their nausea, vomiting, total symptom scores, and number of hospitalizations. Vomiting episodes decreased by 83 percent and 69 percent after both Temp and Perm GES, respectively. Nausea decreased by 62 percent and 46 percent after both Temp and Perm GES, respectively.

The mean symptom scores for the 5 upper GI symptoms associated with CVS, gastric emptying times, and the health related quality of life IDIOMS values at baseline, during Temp GES, and after permanent stimulator implantation for the $11 \mathrm{CVS}$ patients treated with GES, are provided in Table 4.

With temporary stimulation, nausea was reduced from the mean baseline score of 3.55 to 1.35 (62\% reduction), while vomiting was significantly reduced from a mean of 2.7 to 0.45 (83\% reduction). Bloating, early satiety, and total symptom scores were also significantly reduced (Table 4 ). Total symptom scores also showed a reduction from a baseline score of $14.05( \pm 3.23)$ to $4.5( \pm 4.35)$, indicating a $68 \%$ significant reduction after temporary stimulation.

Permanent stimulation resulted in a reduced mean score of 1.9 (46\% reduction) for nausea from baseline values. There were no significant relationships in our small patient sample for gastric emptying times (Table 5), although a significant improvement was seen after both temporary and permanent stimulator implantation for IDIOMS quality of life measures (Table 4). In addition, the total symptom score was reduced to 8.42 ( \pm 5.92$)$, a $40 \%$ improvement when compared to a baseline value of 14.05 ( \pm 3.23$)$.

Vomiting episodes fell from $2.68( \pm 1.27)$ to $0.83( \pm 1.6)$ indicating a significant improvement with permanent stimulation

Table 4. Comparison of Gastrointestinal Symptoms with Gastric Electrical Stimulation Therapy in Cyclic Vomiting Syndrome Patients

\begin{tabular}{|c|c|c|c|c|c|}
\hline & Baseline & Temp GES & $P$-value & Perm GES & $P$-value \\
\hline Nausea & $3.55 \pm 0.69$ & $1.35 \pm 1.70$ & 0.001 & $1.91 \pm 1.11$ & 0.002 \\
\hline Vomiting & $2.68 \pm 1.27$ & $0.45 \pm 1.26$ & 0.001 & $0.83 \pm 1.60$ & 0.019 \\
\hline Bloating & $2.36 \pm 1.52$ & $0.8 \pm 1.32$ & 0.021 & $1.17 \pm 1.60$ & 0.148 \\
\hline Anorexia/early satiety & $2.55 \pm 1.44$ & $0.65 \pm 1.11$ & 0.017 & $1.83 \pm 1.37$ & 0.337 \\
\hline Abdominal pain & $2.90 \pm 1.20$ & $1.35 \pm 1.53$ & 0.003 & $2.67 \pm 1.17$ & 0.694 \\
\hline Total symptom score & $14.05 \pm 3.23$ & $4.5 \pm 4.35$ & 0.001 & $8.42 \pm 5.92$ & 0.021 \\
\hline IDIOMS score (0-30) & $20.0 \pm 4.22$ & $14.45 \pm 4.55$ & 0.003 & $15.22 \pm 5.12$ & 0.006 \\
\hline
\end{tabular}

Temp GES, temporary gastric electrical stimulation; Perm GES, permanent GES; IDIOMS, investigator-derived independent outcome measure scores. 
Table 5. Comparison of Gastric Motility Measures with Gastric Electrical Stimulation Therapy in Cyclic Vomiting Syndrome Patients ${ }^{a}$

\begin{tabular}{lrcccc}
\hline & \multicolumn{1}{c}{ Baseline } & Temp GES & $P$-value & Perm GES & $P$-value \\
\hline GET 1 hr & $63.3 \pm 31.81$ & $76.74 \pm 21.85$ & 0.269 & $51.28 \pm 33.90$ & 0.542 \\
GET 2 hr & $41.30 \pm 28.99$ & $49.87 \pm 29.99$ & 0.524 & $30.08 \pm 24.90$ & 0.511 \\
GET 4 hr & $19.90 \pm 18.08$ & $28.93 \pm 30.09$ & 0.433 & $6.99 \pm 13.98$ & 0.470 \\
\hline
\end{tabular}

${ }^{a}$ Mean gastric emptying test (GET) activity at baseline, post temporary gastric electrical stimulation (Temp GES), and post permanent GES (Perm GES) in patients with cyclic vomiting syndrome measured in $\%$.

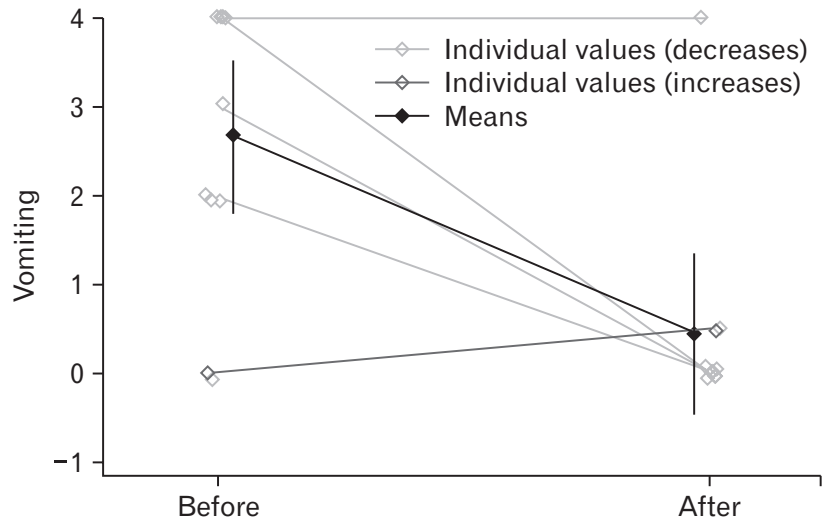

Figure 2. Average change in vomiting scores before and after temporary gastric electrical stimulation (GES). Significant improvements were seen in vomiting scores for all patients with treatment with temporary GES. An average decrease in vomiting score by -2.24 units $(-3.18,-1.29)$ was observed during our study at the end of a 5 -day trial with temporary GES.

(69\% reduction) (Table 4 and Fig. 2). Improvement in vomiting and hospitalization events is also seen at 1-year follow-up after Perm GES therapy. Hospitalizations decreased from 9.14 ( \pm 7.21 ) annual admissions prior to Perm GES to 1.5 ( \pm 1 ) annual admissions thereafter (Table 1). The number of hospital admissions due to vomiting also significantly decreased from 6.19 ( \pm 2.52 ) to 2.25 ( \pm 1.5) after permanent stimulation.

Cutaneous and mucosal electrogram frequencies also changed after temporary electrical stimulation, with the results of electrogastrogram (EGG) and electrogram values for baseline, temporary, and permanent recordings are shown in Table 2 and Figure 1. Baseline cutaneous EGG had a mean frequency of $6.69( \pm 4.57)$ and mean amplitude of $0.12( \pm 0.07)$. After Temp GES there was an insignificant reduction in mean frequency, $5.72( \pm 1.82)$, and amplitude, 0.12 ( \pm 0.09). Further reductions in cutaneous EGG were also seen in frequency, $4.98( \pm 2.21)$, and amplitude, $0.12( \pm$ 0.06), though clinically insignificant. Serosal EGG measurements taken at baseline had a mean frequency of 5.81 ( \pm 2.32$)$ and amplitude of $0.53( \pm 1.09)$. Measurements after Temp GES showed a clinically insignificant reduction in frequency, 4.64 ( \pm 1.55$)$, and amplitude, $0.32( \pm 0.37)$ (Table 2).

\section{Discussion}

The key to the treatment of CVS is its early recognition. Moreover, long-term management of the disorder requires an engaged and responsive collaboration among the doctor(s), patient, and family involved in care, and is ideally treated through the structure of a patient centered medical home. Updated electronic records that can provide access to a patient's stereotypical experiences of CVS, as well as indicate triggers that have pre-disposed the patient to attacks, are particularly useful in an illness that often goes unrecognized and for which emergency departments routinely provide initial treatment. For patients with drug-refractory CVS, however, lessening the impact of the disorder on quality of life may require gastroenterological intervention.

Patients in our pilot study benefitted from both temporary and permanent GES, showing a significant decrease in nausea and vomiting as evidenced by the decreases in symptoms scores seen in Table 4. Our findings demonstrate a $62 \%$ reduction in nausea symptoms from baseline and a concurrent $83 \%$ reduction in vomiting after temporary stimulation. With permanent GES, nausea and vomiting were decreased by $46 \%$ and $69 \%$, respectively. Total symptom scores also significantly decreased from baseline by $68 \%$ and $40 \%$ after Temp GES and Perm GES, respectively. At one-year followup after Perm GES implantation, these patients continued to show significant symptom reduction and decreased number of hospital admissions. Patients' quality of life also improved IDIOMS significantly. There is a possibility that patients' symptoms could have improved without any intervention once their cyclical phase was over, but a significant improvement in hospital admissions after implantation of a Perm GES device was also observed. Patients' burden on healthcare showed improvement with a $66 \%$ and $84 \%$ reduction in hospital admission days from baseline after Temp GES and Perm GES, respectively. In addition, hospital admissions due to vomiting showed a $47 \%$ and $64 \%$ reduction after Temp GES and Perm 
GES, respectively, when compared to baseline values. Thus, given this limited data, we believe that GES provides medically refractory patients with an effective option for relief of this debilitating disease.

Researchers at another center have suggested that GET may be normal or rapid in patients with CVS; ${ }^{19,22,23}$ we observed only that GET showed small improvement after permanent device placement. We believe that acute CVS, which in these patients is sometimes called coalescent CVS, behaves like GP and shares similar pathophysiology. ${ }^{24}$

We will continue to follow our patient cohort's progress long term to learn about the effects of GES on CVS. However, our pilot study results support the use of GES as a treatment option for medically refractory CVS.

Limitations to this study include small sample size, lack of control subjects, non-randomized study, racial differences not included in the data analysis, and recall bias affecting follow up studies. This pilot study was conducted among only 11 patients, so that all results must be seen as preliminary findings. It is also pertinent to note that symptomatic relief from CVS may be secondary to a placebo effect.

In conclusion, GES shows promise as a viable therapeutic option for individuals suffering from drug refractory CVS. Our study demonstrates the potential benefits not only in the patients' quality of life, but also in the overall hospital costs and healthcare burden. Future studies to better understand the pathophysiology of CVS are needed to guide future treatment protocols. In addition, more studies on a larger scale are needed to determine the mechanisms by which GES may exert an effect on CVS. An increase in awareness among physicians in diagnosing CVS, basic science, and clinical research in CVS will hopefully lead to better patient outcomes.

Acknowledgements: Thank you to Catherine McBride at the University of Louisville for manuscript preparation and submission.

\section{Financial support: None.}

Conflicts of interest: Thomas L Abell is licensor, investigator, speaker, and consultant for Medtronic, Inc.

Author contributions: Thomas L Abell: conception and design; Inderpreet Grover, Christopher J Lahr, Danielle C Spree, and Shivangi Kothari: data collection; Inderpreet Grover, Archana Kedar, Richard Kim, and Thomas L Abell: analysis/interpretation of data; Richard Kim and Inderpreet Grover: drafting of the article; Inderpreet Grover and Thomas L Abell: critical revision for important intellectual content; and Inderpreet Grover, David Fleisher, and Thomas L Abell: final approval of the article.

\section{References}

1. Gee S. On fitful or recurrent vomiting. St. Bartholomew's Hospital Rep 1882;18:1-6.

2. Heberden W. Commentaries on the history and causes of diseases. London: Payne and Foss 1806

3. Stickler GB. Relationship between cyclic vomiting syndrome and migraine. Clin Pediatr 2005;44:505-508.

4. Fleisher DR, Gornowicz B, Adams K, Burch R, Feldman EJ. Cyclic vomiting syndrome in 41 adults: the illness, the patients, and problems of management. BMC Med 2005;3:20.

5. Li BU, Misiewicz L. Cyclic vomiting syndrome: a brain-gut disorder. Gastroenterol Clin North Am 2003;32:997-1019.

6. Prakash C, Clouse RE. Cyclic vomiting syndrome in adults: clinical features and response to tricyclic antidepressants. Am J Gastroenterol 1999;94:2855-2860

7. Boles RG, Lovett-Barr MR, Preston A, Li BU, Adams K. Treatment of cyclic vomiting syndrome with co-enzyme Q10 and amitriptyline, a retrospective study. BMC neurology 2010;10:10.

8. Erturk O, Uluduz D, Karaali-Savrun F. Efficacy of nebivolol and amitriptyline in the prophylaxis of cyclic vomiting syndrome: a case report. Neurologist 2010;16:313-314.

9. Vanderhoof JA, Young R, Kaufman SS, Ernst L. Treatment of cyclic vomiting in childhood with erythromycin. J Pediatr Gastroenterol Nutr 1993;17:387-391.

10. Andersen JM, Sugerman KS, Lockhart JR, Weinberg WA. Effective prophylactic therapy for cyclic vomiting syndrome in children using amitriptyline or cyproheptadine. Pediatrics 1997;100:977-981.

11. Van Calcar SC, Harding CO, Wolff JA. L-carnitine administration reduces number of episodes in cyclic vomiting syndrome. Clin Pediatr 2002;41:171-174.

12. Cutts TF, Luo J, Starkebaum W, Rashed H, Abell TL. Is gastric electrical stimulation superior to standard pharmacologic therapy in improving GI symptoms, healthcare resources, and long-term health care benefits? Neurogastroenterol Motil 2005;17:35-43.

13. Anand C, Al-Juburi A, Familoni B, et al. Gastric electrical stimulation is safe and effective: a long-term study in patients with drug-refractory gastroparesis in three regional centers. Digestion 2007;75:83-89.

14. Abell T, Lou J, Tabbaa M, Batista O, Malinowski S, Al-Juburi A. Gastric electrical stimulation for gastroparesis improves nutritional parameters at short, intermediate, and long-term follow-up. JPEN J Parenter Enteral Nutr 2003;27:277-281.

15. Abell TL, Van Cutsem E, Abrahamsson H, et al. Gastric electrical stimulation in intractable symptomatic gastroparesis. Digestion 2002;66:204212.

16. Forster J, Sarosiek I, Lin Z, et al. Further experience with gastric stimulation to treat drug refractory gastroparesis. Am J Surg 2003;186:690-695.

17. Ayinala S, Batista O, Goyal A, et al. Temporary gastric electrical stimulation with orally or PEG-placed electrodes in patients with drug refractory gastroparesis. Gastrointest Endosc 2005;61:455-461.

18. Abell T, McCallum R, Hocking M, et al. Gastric electrical stimulation 
for medically refractory gastroparesis. Gastroenterology 2003;125:421428.

19. Hejazi RA, Lavenbarg TH, McCallum RW. Spectrum of gastric emptying patterns in adult patients with cyclic vomiting syndrome. Neurogastroenterol Motil 2010;22:1298-1302, e338.

20. Tack J, Talley NJ, Camilleri M, et al. Functional gastroduodenal disorders. Gastroenterology 2006;130:1466-1479.

21. Li BU, Lefevre F, Chelimsky GG, et al. North American Society for Pediatric Gastroenterology, Hepatology, and Nutrition consensus statement on the diagnosis and management of cyclic vomiting syndrome. J Pediatr
Gastroenterol Nutr 2008;47:379-393.

22. Pareek N, Fleisher DR, Abell T. Cyclic vomiting syndrome: what a gastroenterologist needs to know. Am J Gastroenterol 2007;102:2832-2840.

23. Turchetti A, Guglielmi S, Fossati C, Matrunola M, Corrado G. Gastric emptying time in cyclic vomiting syndrome in children. Eur Rev Med Pharmacol Sci 2004;8:295-298.

24. O'Grady G, Angeli TR, Du P, et al. Abnormal initiation and conduction of slow-wave activity in gastroparesis, defined by high-resolution electrical mapping. Gastroenterology 2012;143:589-598, e1-e3. 